\title{
Estimation of In Vivo Reticuloendothelial System Phagocytic Activity in Rats by Direct Blood Clearance Techniques and Nuclear Scintigraphy
}

\author{
Deepi G. Goyal, Karol A. Gutowski, and Robert M. Merion \\ Department of Surgery, University of Michigan Medical Center, Ann Arbor
}

\begin{abstract}
There has been considerable Interest in the examination of reticuloendothelial system phagocytic blockade. In this study, the kinetics of phospholipld liposome-mediated and intraperitoneal silica-mediated phagocytic blockade were examined using five methods of analysis of in vivo ${ }^{00 m}$ Tc-labeled albumin clearance and reticuloendothelial cell uptake. Two direct blood sampling techniques revealed significant impairment in ${ }^{0 m}$ Tc-labeled albumin clearance after treatment with silica $(P<0.05)$, while liposome treatment was not associated with such impairment. A method utilizing nuclear scintigraphy for the determination of blood clearance was incapable of detecting sillica-mediated blockade but demonstrated significant impairment by liposomes at $2 \mathrm{hr}(P<0.001), 6 \mathrm{hr}(P<0.05)$, and $24 \mathrm{hr}(P<0.001)$. Gamma camera imaging methods for determination of hepatic uptake demonstrated significant $(P<0.05)$ and reversible impairment of ${ }^{00 m}$ Tc-labeled albumin uptake by liposomes. The most promising of these techniques utilizes deconvolutional analysis of liver region of interest time-activity curves to correct for continuously changing blood concentrations of tracer and for intracellular tracer processing and catabolism. Measurements of reticuloendothelial system phagocytic actlvity should include methods that take into account the observed discrepancies between blood clearance determinations and reticuloendothelial cell uptake.
\end{abstract}

Key words: deconvolutional analysis, reticuloendothelial blockade, silica, liposomes, phospholipid vesicles, endocytosis

\section{INTRODUCTION}

The reticuloendothelial system (RES), consisting of fixed macrophages of the liver, spleen, lungs, and bone marrow, provides the host with a variety of immunological defenses including protection against infection, neoplasia surveillance, and recognition of foreign antigens $[5,17]$. This last function of processing antigens enhances the host's capacity to reject allogeneic tissue and organ transplants, and has stimulated interest in suppression of the RES to modulate the rejection of transplanted organs.

Silica, a macrophage toxin, is one of the best studied agents for inducing RES blockade. Levy and Wheelock have shown that intravenous silica significantly depresses the clearance of colloidal carbon by the RES in treated animals by destroying, rather than saturating RES macrophages [11]. Furthermore, the toxicity observed was specific to the fixed macrophages of the RES, as the number of viable peritoneal macrophages was the same in silica-treated and control mice. Silica was also shown to have indirect effects on host immunity due to interruption of the immune cascade that is initiated by factors released by macrophages. However, the usefulness of silica in studying RES function is limited due to its systematic toxicity, the 2-3 day period required for its effects on RES function, and the fact that its effect may be permanent $[11,12]$.

Liposomes are phospholipid vesicles formed when water-insoluble polar lipids are confronted with water [6]. They can exist in a variety of forms depending on their method of preparation. Reverse phase evaporation vesicles (REVs) are large unilamellar liposomes in which a single lipid layer surrounds a large internal aqueous space. Sonicated unilamellar vesicles (SUVs) are small unilamellar liposomes in which a single lipid layer surrounds a small aqueous space. Multilamellar vesicles (MLVs) are large liposomes consisting of a series of concentric layers of lipid, each separated by an aqueous layer $[8,22]$. As can be inferred, the ratio of lipid to entrapped volume is considerably higher in both MLVs and SUVs than in REVs.

Received July 21, 1989; accepted November 2, 1989.

Reprint requests: Robert M. Merion, Department of Surgery, 2926F Taubman Center, Box 0331, Ann Arbor, MI 48109-0331. 
Initially, liposomes showed promise as a highly specific delivery system for various therapeutic agents. By varying the composition of the phospholipid shell, the vesicles could be targeted for specific tissues. Furthermore, they could be used to deliver a wide variety of agents due to the presence of both aqueous and lipid moieties. Early studies using liposomes, however, demonstrated avid uptake of the vesicles by phagocytic cells of the RES [8, 10, 24]. Kao and Juliano found that an important mechanism of REV removal from the circulation involved uptake by the phagocytic cells of the RES [10]. When animals pretreated with a loading dose of REVs were injected with radiolabeled REVs, the halflife of the test dose particles was extended in the circulation [10]. This is similar to the behavior exhibited by liposomes injected in animals with RES blockade induced by dextran sulphate and carbon [20], suggesting that pretreatment with liposomes induces RES blockade. This blockade appeared to be a result of the saturation of the uptake capacity of the cells of the RES.

Further investigation of the interaction of liposomes with the RES showed that liposome-induced blockade was reversible and was dependent on the type of liposome used [4, 19]. Ellens et al. showed that pretreatment of animals with REVs caused a depression of RES function, as measured by the blood clearance rate of intravenously injected carbon [4]. SUVs, however, did not block RES activity, and the clearance rate of carbon at 1 $\mathrm{hr}$ after injection of the vesicles actually increased. Furthermore, $24 \mathrm{hr}$ after injection of REVs, the rate of carbon clearance returned to control levels, indicating that liposome-mediated blockade of the RES diminished, probably as a result of the vesicles being metabolized by the phagocytes. The difference in clearance rate between REVs and SUVs was attributed to the fact that large liposomes are cleared more quickly than small ones [9]. Thus, the total amount of lipid taken up by the RES after injection of equal doses will be larger with REVs, thereby resulting in greater saturation of phagocytic capacity.

Safe and accurate in vivo measurement of RES phagocytic properties requires the use of a particle that is rapidly removed from vascular circulation and ingested by cells of the RES, that measures $\sim 1 \mu \mathrm{m}$, that has a narrow size distribution, that can be labeled with short-lived radionuclides immediately before use, and that consists of an organic, nonantigenic substance that is metabolizable $[3,18]$. Albumin millimicrospheres have been shown to satisfy the above criteria $[1,7,18]$. Bolognesi et al. found that ${ }^{99 \mathrm{~m}} \mathrm{Tc}$-labeled albumin millimicrospheres form a tracer that is removed by the RES, making it possible to calculate the maximum clearance rate of the RES [1]. Since the macrophages of the liver and spleen manifest approximately 85-95\% of RES ac- tivity, the clearance rate has been taken to be a measure of RES phagocytic function [17].

In the current study, we attempted to determine the kinetics of liposome- and silica-mediated effects on RES phagocytic activity in an in vivo rat model by measuring blood clearance and tissue distribution of a radiolabeled albumin colloid. In particular, we compared five methods of data acquisition and analysis to evaluate quantitative and qualitative differences among methods and to demonstrate whether specific mechanisms of RES phagocytic function affect data obtained by different methods.

\section{MATERIALS AND METHODS Silica}

Quartz silica particles (Sigma) were suspended in saline $(20 \mathrm{mg} / \mathrm{ml})$ and subjected to ultrasonic vibration for $30 \mathrm{~min}$ prior to injection. Injections of $50 \mathrm{mg} / \mathrm{kg}$ or 500 $\mathrm{mg} / \mathrm{kg}$ were administered via the intraperitoneal (i.p.) route 3 days prior to RES measurement.

\section{Liposomes}

REVs were prepared according to the method of Szoka and Papahadjopoulos [22]. Briefly, a 5:4:1 molar ratio of cholesterol (Sigma), phosphatidyl-DL-glycerol (Sigma), and dipalmitoyl-L- $\alpha$-phosphatidyl-DL-choline (Sigma) was dissolved in chloroform and evaporated to a thin dry film in a round-bottomed flask on a rotary evaporator. The film were redissolved in ether, and an aqueous phase of phosphate-buffered saline (PBS) was added. This mixture was then placed in a bath sonicator for $30 \mathrm{~min}$. The resulting homogeneous mixture was reevaporated on the rotary evaporator to achieve a frothy gel followed by a free-running lipid. Doses of $20 \mathrm{mg} / \mathrm{kg}$ were injected intravenously (i.v.) via the tail vein 2,6 , or $24 \mathrm{hr}$ prior to RES measurement. Prepared liposomes were stored, following a nitrogen purge, at $4^{\circ} \mathrm{C}$ for a maximum of 48 $\mathrm{hr}$ before injection.

\section{Animals and Pretreatment Protocols}

Adult male Sprague-Dawley rats (Charles River) weighing 200-400 g were used. The experimental design was approved by the University of Michigan Committee on Use and Care of Animals, and uniform standards of animal care were followed as per the guidelines established by the University of Michigan Unit for Laboratory Animal Medicine. Animals were anesthetized during experimentation with sodium pentobarbital (75 $\mathrm{mg} / \mathrm{kg}$ i.p.). Animals were randomly assigned to six groups. Group 1 served as control and was given $1 \mathrm{ml}$ sterile PBS i.v. via the tail vein, $1 \mathrm{hr}$ prior to measurement of RES function. Animals in groups 2 and 3 were treated 3 days before measurement with silica $50 \mathrm{mg} / \mathrm{kg}$ 
and $500 \mathrm{mg} / \mathrm{kg}$ i.p., respectively. Animals in groups 4, 5 , and 6 were given liposomes at a dose of $20 \mathrm{mg} / \mathrm{kg}$ i.v. at 2, 6, and $24 \mathrm{hr}$ before RES measurement, respectively.

\section{Measurement of RES Activity}

Blood clearance and tissue distribution of ${ }^{99 \mathrm{~m}} \mathrm{Tc}$-labeled colloidal albumin (Microlite, DuPont) were used as monitors of RES phagocyte function. Following injection of $1 \mathrm{mCi}$ of the tracer via the penile vein, $50 \mu \mathrm{l}$ blood samples were drawn from the tail vein at $1 \mathrm{~min}$ intervals for $10 \mathrm{~min}$ and at 12 and $15 \mathrm{~min}$ after injection. Simultaneously, images were acquired by a gamma camera (pinhole collimator with magnification) at $30 \mathrm{sec}$ intervals from $30 \mathrm{sec}$ preinjection to $14 \mathrm{~min}$ postinjection.

Five different methods were employed to analyze the data. Methods 1 and 2 used blood clearance data. Samples were counted in a Packard Autogamma Scintillation Spectrometer. Method 1 incorporated a log-linear regression by the least squares method, resulting in a biexponential function. The fast, early portion was stripped of the late, slow portion of the plot to obtain the net slope or clearance index $(\mathrm{K})$ of the regression line from 2 to 5 $\min [12]$. Method 2 utilized a determination of the simple slope of a semilogarithmic plot of blood counts between 2 and $7 \mathrm{~min}$ after injection [7].

Methods 3 through 5 utilized data obtained from the gamma camera. Regions of interest were traced over the liver and heart, and time-activity curves were processed for each region of interest. Method 3 applied a log-linear regression by least squares method on the data from the gamma camera-generated liver region of interest counts between 2 and $7 \mathrm{~min}$ after injection to obtain a liver uptake index (K) [7]. In method 4, the slope of a semilogarithmic plot of the counts between 75 and 315 seconds from the heart region of interest was used to approximate clearance of tracer from the blood (as in method 1). Method 5 utilized a deconvolutional analysis of gamma camera images over the liver region of interest [23]. This technique allows for the measurement of hepatic functions by simulating a bolus injection of the tracer directly into the afferent blood supply of the liver and corrects for the changing concentration of tracer in the blood as it is extracted with each pass through the liver.

\section{Statistical Analysis}

Descriptive data are expressed as mean \pm SEM. Slopes obtained by the various methods of analysis were compared by the Kolmogorov-Smirnov two sample test, using a $P$ value less than 0.05 as the minimum level of statistical significance.

\section{RESULTS}

The results of the five methods of data acquisition and analysis in the six experimental groups are shown in Table 1. Using method 1, high-dose silica administration (group 3) resulted in a significant reduction in the rate of blood clearance of the albumin colloid compared with PBS-treated controls (group 1) $(P=0.023)$. The low dose of silica (group 2) was associated with a reduction in blood clearance compared with controls, but there was a larger range of values obtained in group 2 , and the difference from control values did not achieve statistical significance. Liposomes administered 2, 6, and $24 \mathrm{hr}$ (groups 3, 4, and 5, respectively) prior to determinations by method 1 failed to demonstrate a significant reduction of the clearance rate of radiolabeled albumin colloid from blood.

Using method 2, in which analysis of blood clearance data was restricted to the period of time between 2 and 7 min after injection of the colloid tracer, both silica doses resulted in significant reduction of RES ability to clear blood of the albumin marker $(P<0.001)$. The low dose of silica (group 2) was significantly more effective in reducing blood clearance than the high dose (group 3$)(P$ $=0.023$ ). Similar to method 1 , liposome administration did not appear to affect blood clearance significantly as measured by method 2 . Both doses of silica produced significantly more impairment of blood clearance capability than liposomes did at any time $(P=0.002)$ (Table 1).

In general, the gamma camera imaging acquisition and analysis techniques produced more intragroup variability than the direct blood clearance techniques (Table 1). Method 3, which monitored hepatic uptake of tracer (as opposed to blood clearance) was relatively insensitive to the effects of the low dose of silica. The high dose of silica actually resulted in a significantly enhanced hepatic uptake of albumin tracer $72 \mathrm{hr}$ after administration, whereas uptake by the liver was significantly impaired 2 $\mathrm{hr}$ after administration of liposomes $(P=0.023)$. This impairment by liposomes was reversed by $6 \mathrm{hr}(P=$ 0.002 vs. $2 \mathrm{hr}$ ), and by $24 \mathrm{hr}$ hepatic uptake was increased over control animals in group 1, although the effect did not quite achieve statistical significance $(P=$ 0.066 ) because of marked interanimal variability in group 6 (Table 1).

Method 4 utilized gamma camera measurements of blood clearance as determined by changes in counts obtained in a region of interest drawn over the heart. No significant effect of silica at either dose compared with control could be demonstrated by this method, whereas liposomes administered at each of the three time points prior to testing resulted in significant reduction in blood clearance of the albumin tracer by gamma camera deter- 
TABLE 1. Reticuloendothelial System Phagocytic Indices Measured by Five Methods of Analysis"

\begin{tabular}{|c|c|c|c|c|c|c|c|c|c|c|c|c|c|}
\hline \multirow{2}{*}{$\begin{array}{l}\text { Method of } \\
\text { analysis }\end{array}$} & \multirow{2}{*}{ Group } & \multirow[b]{2}{*}{ Agent } & \multirow{2}{*}{$\begin{array}{c}\text { Dose } \\
\text { and route }\end{array}$} & \multirow{2}{*}{$\begin{array}{c}\text { Time before } \\
\text { measurement } \\
\text { (hr) }\end{array}$} & \multirow[b]{2}{*}{ No. } & \multirow[b]{2}{*}{ Mean \pm SEM } & \multirow[b]{2}{*}{ Range } & \multicolumn{6}{|c|}{$P$ vs. group number } \\
\hline & & & & & & & & 1 & 2 & 3 & 4 & 5 & 6 \\
\hline 1 & 1 & PBS & $1 \mathrm{ml}$ i.v. & 1 & 15 & $0.534 \pm 0.027$ & $0.402-0.676$ & 1.000 & 0.162 & 0.023 & 0.613 & 0.613 & 0.613 \\
\hline 1 & 2 & Silica & 50 mg/kg i.p. & 72 & 8 & $0.473 \pm 0.027$ & $0.332-0.570$ & & 1.000 & 0.613 & 0.162 & 0.162 & 0.613 \\
\hline 1 & 3 & Silica & $500 \mathrm{mg} / \mathrm{kg}$ i.p. & 72 & 8 & $0.492 \pm 0.012$ & $0.435-0.532$ & & & 1.000 & 0.023 & 0.023 & 0.613 \\
\hline 1 & 4 & Liposomes & $20 \mathrm{mg} / \mathrm{kg}$ i.v. & 2 & 8 & $0.505 \pm 0.022$ & $0.390-0.558$ & & & & 1.000 & 0.162 & 0.162 \\
\hline 1 & 5 & Liposomes & $20 \mathrm{mg} / \mathrm{kg}$ i.v. & 6 & 8 & $0.523 \pm 0.018$ & $0.467-0.580$ & & & & & 1.000 & 0.613 \\
\hline 1 & 6 & Liposomes & $20 \mathrm{mg} / \mathrm{kg}$ i.v. & 24 & 8 & $0.509 \pm 0.018$ & $0.473-0.617$ & & & & & & 1.000 \\
\hline 2 & 1 & PBS & $1 \mathrm{ml} \mathrm{i.v.}$ & 1 & 15 & $0.168 \pm 0.004$ & $0.131-0.192$ & 1.000 & 0.000 & 0.000 & 0.613 & 0.613 & 0.613 \\
\hline 2 & 2 & Silica & $50 \mathrm{mg} / \mathrm{kg}$ i.p. & 72 & 8 & $0.130 \pm 0.009$ & $0.082-0.163$ & & 1.000 & 0.023 & 0.002 & 0.002 & 0.002 \\
\hline 2 & 3 & Silica & $500 \mathrm{mg} / \mathrm{kg}$ i.p. & 72 & 8 & $0.141 \pm 0.006$ & $0.112-0.168$ & & & 1.000 & 0.002 & 0.002 & 0.002 \\
\hline 2 & 4 & Liposomes & $20 \mathrm{mg} / \mathrm{kg}$ i.v. & 2 & 8 & $0.161 \pm 0.008$ & $0.114-0.179$ & & & & 1.000 & 0.998 & 0.162 \\
\hline 2 & 5 & Liposomes & $20 \mathrm{mg} / \mathrm{kg}$ i.v. & 6 & 8 & $0.164 \pm 0.005$ & $0.144-0.177$ & & & & & 1.000 & 0.613 \\
\hline 2 & 6 & Liposomes & $20 \mathrm{mg} / \mathrm{kg}$ i.v. & 24 & 8 & $0.161 \pm 0.007$ & $0.134-0.189$ & & & & & & 1.000 \\
\hline 3 & 1 & PBS & $1 \mathrm{ml}$ i.v. & 1 & 15 & $-0.020 \pm 0.002$ & $-0.038--0.011$ & 1.000 & 0.613 & 0.023 & 0.023 & 0.162 & 0.066 \\
\hline 3 & 2 & Silica & 50 mg/kg i.p. & 72 & 8 & $-0.023 \pm 0.008$ & $-0.068--0.002$ & & 1.000 & 0.162 & 0.023 & 0.162 & 0.985 \\
\hline 3 & 3 & Silica & $500 \mathrm{mg} / \mathrm{kg}$ i.p. & 72 & 8 & $-0.013 \pm 0.005$ & $-0.041--0.002$ & & & 1.000 & 0.002 & 0.023 & 0.985 \\
\hline 3 & 4 & Liposomes & $20 \mathrm{mg} / \mathrm{kg}$ i.v. & 2 & 8 & $-0.023 \pm 0.002$ & $-0.033--0.013$ & & & & 1.000 & 0.002 & 0.000 \\
\hline 3 & 5 & Liposomes & $20 \mathrm{mg} / \mathrm{kg}$ i.v. & 6 & 8 & $-0.014 \pm 0.002$ & $-0.026--0.009$ & & & & & 1.000 & 0.423 \\
\hline 3 & 6 & Liposomes & $20 \mathrm{mg} / \mathrm{kg}$ i.v. & 24 & 6 & $-0.015 \pm 0.011$ & $-0.011--0.017$ & & & & & & 1.000 \\
\hline 4 & 1 & PBS & $1 \mathrm{ml}$ i.v. & 1 & 14 & $2.601 \pm 0.081$ & $2.003-2.955$ & 1.000 & 0.162 & 0.162 & 0.000 & 0.023 & 0.000 \\
\hline 4 & 2 & Silica & $50 \mathrm{mg} / \mathrm{kg}$ i.p. & 72 & 8 & $2.668 \pm 0.228$ & $2.008-4.050$ & & 1.000 & 0.023 & 0.000 & 0.162 & 0.011 \\
\hline 4 & 3 & Silica & $500 \mathrm{mg} / \mathrm{kg}$ i.p. & 72 & 8 & $2.575 \pm 0.065$ & $2.337-2.912$ & & & 1.000 & 0.000 & 0.023 & 0.000 \\
\hline 4 & 4 & Liposomes & $20 \mathrm{mg} / \mathrm{kg}$ i.v. & 2 & 8 & $3.102 \pm 0.069$ & $2.761-3.375$ & & & & 1.000 & 0.023 & 0.526 \\
\hline 4 & 5 & Liposomes & 20 mg/kg i.v. & 6 & 8 & $2.974 \pm 0.451$ & $1.311-5.671$ & & & & & 1.000 & 0.111 \\
\hline 4 & 6 & Liposomes & $20 \mathrm{mg} / \mathrm{kg}$ i.v. & 24 & 7 & $3.063 \pm 0.107$ & $2.529-3.419$ & & & & & & 1.000 \\
\hline 5 & 1 & PBS & $1 \mathrm{ml}$ i.v. & 1 & 14 & $-0.051 \pm 0.007$ & $-0.103--0.027$ & 1.000 & 0.613 & 0.613 & 0.002 & 0.023 & 0.526 \\
\hline 5 & 2 & Silica & $50 \mathrm{mg} / \mathrm{kg}$ i.p. & 72 & 8 & $-0.074 \pm 0.022$ & $-0.182--0.017$ & & 1.000 & 0.162 & 0.023 & 0.613 & 0.526 \\
\hline 5 & 3 & Silica & $500 \mathrm{mg} / \mathrm{kg}$ i.p. & 72 & 8 & $-0.058 \pm 0.004$ & $-0.071--0.041$ & & & 1.000 & 0.000 & 0.002 & 0.011 \\
\hline 5 & 4 & Liposomes & $20 \mathrm{mg} / \mathrm{kg}$ i.v. & 2 & 8 & $-0.038 \pm 0.004$ & $-0.060--0.021$ & & & & 1.000 & 0.002 & 0.111 \\
\hline 5 & 5 & Liposomes & $20 \mathrm{mg} / \mathrm{kg}$ i.v. & 6 & 8 & $-0.062 \pm 0.011$ & $-0.132--0.041$ & & & & & 1.000 & 0.526 \\
\hline 5 & 6 & Liposomes & $20 \mathrm{mg} / \mathrm{kg}$ i.v. & 24 & 7 & $-0.047 \pm 0.006$ & $-0.067--0.020$ & & & & & & 1.000 \\
\hline
\end{tabular}

asee text for description of data acquisition and analysis methods.

mination. This contrasts to the results obtained by the two direct blood sampling techniques (methods 1 and 2), where no such effect of liposomes was seen (Table 1). Some of this difference may be a result of inclusion of left lung tissue in the gamma camera region of interest.

The final method of analysis utilized deconvolutional analysis of hepatic region of interest curves generated by method 3, adjusting for the continuously changing blood concentration of tracer that is presented to the liver. Using method 5, silica effects on hepatic uptake were seen, but did not achieve statistical significance. Hepatic uptake was significantly increased $2 \mathrm{hr}$ after liposome administration $(P=0.002)$, but by $6 \mathrm{hr}$ significant reduction in hepatic extraction was demonstrable $(P=$ 0.023). This contrasts to the uncorrected results obtained by method 3 , which showed earlier impairment of hepatic extraction and more rapid recovery. By $24 \mathrm{hr}$ after liposome treatment, hepatic albumin tracer extraction returned to control values when measured by the deconvolutional analysis technique.

\section{DISCUSSION}

The process of macrophage phagocytosis has been described in terms of three distinct steps [16]. The initial interaction of foreign particulate or antigenic material consists of association of the particle with a macrophage membrane receptor. This receptor may be specific or nonspecific. Following adsorption, a surface-initiated process of engulfment ensues, generally by endocytosis [13]. Finally, once internalized, intralysosomal degradation of most foreign particles takes place [16].

Normann has described the saturation kinetics of the initial interaction with the macrophage membrane receptor as being limited by the formation of an intermediate colloid-membrane complex prior to internalization [13]. It appears that one type of colloid can competitively inhibit the interaction of another colloid with the membrane, similar to competitive substrate inhibition in enzyme kinetics [14]. Two postulated mechanisms for competitive inhibition include direct occupation of the 
membrane site preempting interaction by a second colloid moiety, or competition for opsonic molecules [13]. The latter appears to be unlikely in the face of evidence that depletion of opsonins was not observed during liposome-mediated RES blockade [4]. In other studies, dissimilar particulate substances such as lipid emulsions, iron, denatured albumin, and rabbit red blood cells were capable of inhibiting phagocytosis of one another [14]. This implies that most substances undergo phagocytosis through a process with at least some common pathways. Finally, the observation has been made that impairment of blood clearance is not necessarily attributable to dysfunction of the RES itself. The relative amounts of the phagocytic blocking agent and the labeled test substance may be an important factor in the appearance of blockade by affecting access to the surface colloid-membrane complex. By altering the ratio of putative blocking substance to test substance, RES blockade could be made to appear or disappear, further substantiating the concept of competition at the level of the colloid-membrane complex [15].

The effects of silica on macrophage function have been well studied $[11,12]$. As a direct toxin, intravenous silica has been found to depress hepatic Kupffer cell function profoundly, while peritoneal macrophages may be less severely affected [11]. In previous studies, we showed that intraperitoneal administration of silica resulted in significant reduction of the clearance of ${ }^{99 \mathrm{~m}} \mathrm{Tc}$ labeled albumin millimicrospheres as well as decreased hepatic uptake. These findings were again seen in the current study, which utilized the more readily available ${ }^{99 \mathrm{~m}} \mathrm{Tc}$-labeled albumin colloid as a test substance. Using two direct sampling blood clearance methods, high- and low-dose silica resulted in a markedly reduced rate of clearance of the radiolabeled albumin tracer from the circulation. Unlike tissue distribution methods, which rely upon total organ test substance uptake at a fixed time point, the gamma camera imaging analysis used in method 3 measured the dynamic appearance of radioactivity over the liver region of interest over a defined interval of time. The slope of the liver region of interest curve was used to generate a liver uptake index. Lowdose silica did not produce significant reduction of the liver uptake index, while high-dose silica actually resulted in a slight but significant enhancement of hepatic uptake. These findings may reflect membrane effects of silica. Although blood clearance is impaired because of membrane alterations produced by the silica, radiolabeled albumin that successfully interacts at the colloidmembrane interface may be phagocytosed at a more normal rate. Given these findings, it is difficult to explain the discrepant results using analysis of gamma camera images over the heart region of interest (method 4). No impairment of clearance was seen using this method, although the marked variation in results among the animals in this group leave open the possibility that the failure to demonstrate significant blockade represents a type II error. Method 5, which used deconvolutional analysis of the liver region of interest images, also failed to show significant blockade with silica. This technique corrects the liver time-activity curve for confounding factors such as the changing blood concentration of tracer presented to the liver for phagocytosis and the contribution of postphagocytic tracer processing and recirculation [23]. In contrast to method 3 , which is uncorrected for these factors, method 5 showed reduction of the liver deconvolution index for both dose levels of silica compared with controls. Although these differences did not achieve statistical significance, they suggest that silica may affect macrophage-mediated particle engulfment and internalization in addition to impairing blood clearance by interfering with the rate-limiting interaction of the test substance with the cell membrane.

Studies of the uptake and processing of liposomal phospholipids by phagocytic cells have been previously reported $[2,21]$. Straubinger et al. have shown that negatively charged liposomes are endocytosed by coated pits in a manner analogous to other molecules that interact with surface receptors [21]. Such liposomes appear to associate rapidly with cells, saturating at approximately $10^{4}$ liposomes per cell. Once internalized, phospholipids are degraded in isolated Kupffer cells [2]. It has been suggested that acidification of the endocytosed vesicle, rather than liposome fusion, results in the release of liposomal contents into the cytoplasm [21].

Kao and Juliano [10] made the important observation that the RES might not constitute the only site or mechanism of liposomal clearance. As evidence for this assertion, they showed that despite a substantial alteration in the clearance rate of REVs after administration of latex beads, there was not a major change in the tissue distribution of the radiolabeled REVs. These studies did not, however, specifically address the issue of phagocytic rate, since saturation of the membrane interaction producing reduced blood clearance might still allow for the ultimate accumulation of control levels of liposomes during the $2 \mathrm{hr}$ incubation interval studied. Souhami et al. [20] carefully studied the effect of blockade by dextran sulphate and carbon on liposome clearance and tissue distribution. In contrast to Kao and Juliano's work, they found that blockade reduced both liposome clearance and hepatic uptake, except in the cases of positively charged large liposomes and neutral small unilamellar vesicles, in which blockade produced delayed clearance without altered liver uptake. These authors have postulated that, in addition to Kupffer cells, other cells in the liver such as sinusoidal endothelial cells and hepatocytes must be capable of liposome uptake. 
Previous studies have shown that liposomes are capable of blocking the RES [12], in some cases reversibly [4]. The half-life of REVs after intravenous injection is approximately $40 \mathrm{~min} \mathrm{[10],} \mathrm{so} \mathrm{that} \mathrm{by} \mathrm{the} \mathrm{time} \mathrm{the} \mathrm{RES}$ studies are performed 2,6 , or $24 \mathrm{hr}$ later the vast majority of liposomes will have disappeared from circulation. In one study, REVs produced significantly reduced blood clearance of ${ }^{{ }^{99} \mathrm{~m}} \mathrm{Tc}$-labeled albumin millimicrospheres for at least $18 \mathrm{hr}$ after liposome administration [12]. Ellens et al. [4] showed that the RES blockade present $4 \mathrm{hr}$ after dosing with liposomes was reversed by $24 \mathrm{hr}$. In the current study, REVs showed only modestly reduced clearance by direct blood sampling techniques. Measurements by gamma camera imaging over the heart region of interest, however, demonstrated significant impairment of tracer clearance as early as $2 \mathrm{hr}$ after liposome treatment, which persisted at $24 \mathrm{hr}$. Hepatic uptake rates, in contrast to static tissue distribution studies, yielded interesting results. Method 3 (crude hepatic uptake index) appeared to demonstrate significant impairment of hepatic RES function $2 \mathrm{hr}$ after liposome administration, with recovery to control values at 6 and $24 \mathrm{hr}$.

The deconvolutional analysis technique utilized in method 5 to evaluate gamma camera-generated hepatic uptake yielded the clearest picture. This technique continuously corrects for the changing blood concentration of the tracer substance that is presented to the hepatic RES cells, allowing for more precise estimation of hepatic RES kinetics. In this way, confounding factors such as recirculation of tracer, changes in the intracellular metabolic processing of radiolabeled colloid, and the release of free ${ }^{99 \mathrm{~m}} \mathrm{Tc}$ after degradation of the colloid are accounted for [23]. Additionally, prior studies of organ distribution analysis after administration of ${ }^{99 \mathrm{~m}} \mathrm{Tc}$ labeled albumin have shown an 80-100-fold greater uptake of tracer in the liver when compared with the spleen or lung, reflecting the proportionally greater concentration of RES activity in the liver [18].

Using the deconvolutional technique (method 5), we have demonstrated a statistically significant enhancement of hepatic RES function $2 \mathrm{hr}$ after liposome administration $(P=0.002$ vs. control), significant blockade by $6 \mathrm{hr}(P=0.023$ vs. control; $P=0.002$ vs. $2 \mathrm{hr}$ liposomes), and a return to baseline levels at $24 \mathrm{hr}$. These results suggest that the process of liposomal phospholipid-membrane complex interaction may transiently increase overall phagocytic activity in the liver. Once saturation of membrane receptors occurs, however, sufficient quantities of liposomes may be associated with the cell to impede this step in the phagocytic process. Further, when a large enough quantity of endocytosed lipid is present in the cell, the engulfment function may also be overwhelmed until lipid metabolism and degradation occurs. Finally, after sufficient time has passed, the cell is again capable of phagocytosis at a normal rate by the sequential process of association with the membrane receptor followed by endocytic engulfment.

In conclusion, the data presented in this study indicate that the method of analysis is a crucial factor in the accurate estimation of RES function. A combination of blood clearance and hepatic RES uptake analysis using the technique of deconvolutional analysis appears to offer the clearest picture of the dynamic process of in vivo RES-mediated phagocytic function. This technique avoids the limitations associated with the isolated use of simple blood clearance techniques and the methodological shortcomings of pure time-activity curve generation. Finally, this technique has the potential to offer new insights into the in vivo association, uptake, and processing of liposomes that are capable of reversible blockade of hepatic RES phagocytic function.

\section{ACKNOWLEDGMENTS}

We gratefully acknowledge the technical assistance of Sarah Warber-Matich and Steve Pitt and the technical advice of Jack Juni, MD.

\section{REFERENCES}

1. Bolognesi, M., Merkel, C., Gatta, A. The use of human albumin millimicrospheres tagged with ${ }^{99 \mathrm{~m}} \mathrm{Tc}$ in the evaluation of the removal capacity of the reticuloendothelial system. Eur. J. Nucl. Med. 13, 254, 1987.

2. Dijkstra, J., Van Galen, M., Regts, D., Scherphof, G. Uptake and processing of liposomal phospholipids by Kupffer cells in vitro. Eur. J. Biochem. 148, 391, 1985.

3. Dobson, E.L., Hardin, B.J. The behavior of intravenously injected particulate material. Acta Med. Scand. 273 (Suppl), 1, 1952.

4. Ellens, H., Mayhew, E., Rustum, Y.M. Reversible depression of the reticuloendothelial system by liposomes. Biochim. Biophys. Acta 714, 479, 1982.

5. Gilbreath, M.J., Nacy, C.A., Hoover, D.L., Alving, C.R., Swartz, G.M., Meltzer, M.S. Macrophage activation for microbicidal activity against Leishmania Major: Inhibition of lymphokine activation by phosphatidylcholine-phosphatidylserine liposomes. J Immunol 134, 6420, 1985.

6. Gregoriadis, G. The carrier potential of liposomes in biology and medicine. N. Engl. J. Med. 295, 704 and 765, 1976.

7. Holmberg, S.B., Hafstrom, L., Jacobsson, L. Phagocytosis and dynamic RES scintigraphy: An evaluation of commercial colloids in rats. Nucl. Med. Commun. 8, 335, 1987.

8. Hsu, M.J., Juliano, R.L. Interactions of liposomes with the reticuloendothelial system. II: Nonspecific and receptor-mediated uptake of liposomes by mouse peritoneal macrophages. Biochim. Biophys. Acta 720, 411, 1982.

9. Juliano, R.L., Stamp, D. The effect of particle size and charge on the clearance rates of liposomes and liposome encapsulated drugs. Biochem. Biophys. Res. Commun. 63, 651, 1975.

10. Kao, Y.J., Juliano, R.L. Interactions of liposomes with the reticuloendothelial system: Effect of reticuloendothelial blockade 
on the clearance of large unilamellar vesicles. Biochim. Biophys. Acta 677, 453, 1982.

11. Levy, M.H., Wheelock, E.F. Effects of intravenous silica on immune and nonimmune functions of the murine host. J. Immunol. $115,41,1975$.

12. Merion, R.M. Measurement of reticuloendothelial system phagocytic activity in the rat after treatment with silica, liposomes, and cyclosporine. Transplantation 40, 86, 1985.

13. Normann, S.J. The kinetics of phagocytosis: I. A study on the clearance of denatured bovine albumin and its competitive inhibition by denatured human albumin. J. Reticuloendothel. Soc. 14, 587, 1973.

14. Normann, S.J. The kinetics of phagocytosis: II. Analysis of in vivo clearance with demonstration of competitive inhibition between similar and dissimilar foreign particles. Lab. Invest. 31, $161,1974$.

15. Normann, S.J. The kinetics of phagocytosis: III. Two colloid reactions, competitive inhibition, and degree of inhibition between similar and dissimilar foreign particles. Lab. Invest. 31, 286, 1974.

16. Reska, S.N., Vyska, K., Feinendegen, L.E. In vivo assessment of phagocytic properties of Kupffer cells. J. Nucl. Med. 22, 405, 1981.

17. Saba, T.M. Physiology and physiopathology of the reticuloendothelial system. Arch. Int. Med. 126, $1031,1981$.
18. Scheffel, U., Rhodes, B.A., Natarajan, T.K., Wagner, H.N. Albumin microspheres for study of the reticuloendothelial system. J. Nucl. Med. 13, 498, 1972.

19. Schwendener, R.A., Lagocki, P.A., Rahman, Y.E. The effects of charge and size on the interaction of unilamellar liposomes with macrophages. Biochim. Biophys. Acta 772, 93, 1984.

20. Souhami, R.L., Patel, H.M., Ryman, B.E. The effect of reticuloendothelial blockade on the blood clearance and tissue distribution of liposomes. Biochim. Biophys. Acta 674, 354, 1981.

21. Straubinger, R.M., Hong, K., Friend, D.S., Papahadjopoulos, D. Endocytosis of liposomes and intracellular fate of encapsulated molecules: Encounter with a low pH compartment after internalization in coated vesicles. Cell 32, 1069, 1983.

22. Szoka, F., Papahadjopoulos, D. Procedure for preparation of liposomes with large aqueous space and high capture by reversephase evaporation. Proc. Natl. Acad. Sci. USA 75, 4194, 1978.

23. Tagge, E., Campbell, D.A., Reichle, R., Averill, D.A., Merion, R.M., Dafoe, D.C., Turcotte, J.G., Juni, J.E. Quantitative scintigraphy with deconvolutional analysis for the dynamic measurement of hepatic function. J. Surg. Res. 42, 605, 1987.

24. Wassef, N.M., Roerdink, F., Richardson, E.C., Alving, C.R. Suppression of phagocytic function and phospholipid metabolism in macrophages by phosphatidylinositol liposomes. Proc. Natl. Acad. Sci. USA 81, 2655, 1984. 